\title{
Psychiatric aspects of diabetes mellitus
}

\author{
Anne M. Doherty
}

\begin{abstract}
SUMMARY
Diabetes is an increasingly common health problem, especially in the West, where there is an emerging epidemic of type 2 diabetes, closely related to the epidemic of obesity. Many people with diabetes struggle to optimise their diabetes control, often because they also have mental illnesses or psychological and social problems. Poor diabetes control has significant consequences for the individual, and if not addressed will result in complications that include blindness, kidney failure and even amputations. There are also consequences for health services resulting from increased admissions and emergency department presentations with diabetes-related difficulties. In the long-term, the costs associated with complications such as renal failure and amputation are high. Addressing the psychiatric and psychological barriers to good glucose control can help reduce the burden of diabetes and its complications on both the individual and the health service.
\end{abstract}

\section{LEARNING OBJECTIVES}

- Understand the relationship between common mental health problems and diabetes

- Understand psychological barriers to diabetes self-care

- Consider the role of the psychiatrist in closing the mortality gap in diabetes and other longterm conditions between those with and without severe mental illness

\section{DECLARATION OF INTEREST}

None

Diabetes mellitus is a long-term condition characterised by elevated blood glucose, which unchecked can result in serious complications due to the effects of glucotoxicity. It is a common condition that is rising in incidence and prevalence. In the UK it has a current prevalence of $6 \%$ : 3.2 million people have a diagnosis of diabetes, and almost three-quarters of a million more are likely to have undiagnosed diabetes (Quality and Outcomes Framework data for 2012-2013, cited in Diabetes UK 2014: p. 3). Type 2 is the most common form of diabetes, with only $10-15 \%$ of cases attributable to type 1 diabetes and other less common forms.

Approximately a third of people with diabetes have psychological and/or social problems that interfere with their ability to manage their diabetes (Grigsby 2002). This leads to poorly controlled blood glucose levels, increased risk of diabetes-related complications and premature mortality, poorer quality of life and increased cost to the individual and the health service.

\section{Types of diabetes}

\section{Type 1 diabetes}

Type 1 diabetes is an autoimmune condition that destroys the insulin-producing $\beta$-cells in the pancreas. As insulin is essential in the regulation of blood glucose levels, a deficiency of insulin results in elevated glucose levels.

\section{Type 2 diabetes}

Type 2 diabetes has a rather more complex aetiology. It includes different degrees of insulin deficiency and insulin resistance, but the individual continues to produce some endogenous insulin, especially in the earlier phases of the condition. Insulin deficiency may be due to failure of the pancreatic $\beta$-cells. Insulin resistance is predicted by abdominal obesity and results in cells being unable to take up blood glucose, resulting in hyperglycaemia.

\section{Some other types}

\section{Gestational diabetes}

This occurs in women without a history of diabetes who develop hyperglycaemia in pregnancy. It affects about $4 \%$ of pregnant women, and in some cases may precede the development of type 2 diabetes. It requires strict control to avoid the diabetic complications of pregnancy for the neonate (National Institute for Health and Care Excellence (NICE) 2008a).

\section{Maturity-onset diabetes of the young (MODY)}

MODY is caused by autosomal dominant monogenetic defects of $\beta$-cell function, with

\section{ARTICLE}

Anne Doherty is a consultant liaison psychiatrist leading the 3 Dimensions of Care for Diabetes (3DFD) service at King's College Hospital, London. This service won a BMJ award in 2014 for improving outcomes in poorly controlled diabetes by providing integrated psychiatric, psychological and social care. Her research interests are in adjustment disorder and the overlap of medical and psychiatric disorders. Correspondence Dr Anne M. Doherty, King's College Hospital, Denmark Hill, London SE5 9RS, UK. Email: anne.doherty@kcl.ac.uk 
resultant impaired insulin secretion expressed as mild hyperglycaemia at a young age. It is very rare: 50 cases per million (Kropff 2011).

Latent autoimmune diabetes in adults (LADA)

LADA is a rare form of adult-onset diabetes with islet antibodies at the early stage, but only slow progression of autoimmune $\beta$-cell failure (Stenström 2005).

\section{Secondary diabetes}

Secondary diabetes is caused by a wide variety of conditions, including pancreatitis, medications, surgery, Cushing's syndrome and cystic fibrosis, and may account for as many as $10 \%$ of cases of diabetes (Ewald 2012).

\section{The management of diabetes}

In type 1 diabetes the most effective treatments are those that attempt to replicate the natural pattern of insulin production - a background stream of basal insulin with boluses to reduce postprandial glucose peaks. This may take the form of a basal-bolus injection regimen, where the individual injects basal or slow-release insulin once or twice a day and boluses of quick-acting insulin with each meal. Another option is insulin pump therapy (continuous subcutaneous insulin infusion), where the individual wears a pump that provides a steady infusion of quick-acting insulin, with boluses controlled by the person for meals. Finally, there is a fixed-dose insulin regimen, where control of glucose levels is achieved by strict control of carbohydrate consumption at each meal: this regimen is less popular. Recent educational innovations such as 'dose adjustment for normal eating' (DAFNE) allow the individual more control over their diabetes and more choice in their diet, but increase the complexity of diabetes self-management.

The management of type 2 diabetes requires different treatments at different stages of the condition (NICE 2008b). In the early stages, diabetes can be controlled by lifestyle modification. However, many patients require oral medications, including metformin (which is first line for people with normal renal function), the sulfonylureas, thiazolidinediones, dipeptidyl peptidase-4 (DPP4) inhibitors (gliptins) and, more recently, sodium/ glucose co-transporter-2 (SGLT-2) inhibitors. For those who do not respond adequately to oral hypoglycaemic agents, there are injectable options: glucagon-like peptide-1 (GLP-1) and insulin. GLP-1 is an anorexigenic peptide produced centrally in the nucleus tractus solitarius of the medulla oblongata and peripherally in the pancreas and gastrointestinal tract. People with diabetes have been observed to lose body weight on GLP-1 analogue treatment, and it has been hypothesised that this may be due to its central effect on the mesolimbic system (van Bloemendaal 2014).

The cornerstones of diabetes self-management are education, diet, monitoring, lifestyle and adherence to treatments. Maintaining these selfmanagement measures requires a high degree of motivation, and many patients find them onerous, especially if they also have mental health problems, social problems or life events to deal with.

\section{The importance of glycaemic control}

Diabetes is associated with serious complications that are the consequences of glucotoxicity. These include neuropathy, maculopathy, renal failure and amputations. The risk of developing these complications is increased by poor glycaemic control. Glycated haemoglobin or $\mathrm{HbA}_{1 \mathrm{c}}$ is an objective measure of overall glycaemic control that allows targets to be set.

The UK Prospective Diabetes Study (UKPDS) was a large study following over 5000 people with type 2 diabetes for 10 years (www.dtu.ox.ac.uk/ ukpds). One of the papers published from this study reported that intensive glycaemic control was associated with a reduction in 'diabetesrelated endpoints' (e.g. sudden death, renal failure, amputation, blindness in one eye) (UKPDS 1998). Another reported that a $1 \%$ reduction in $\mathrm{HbA}_{1 \mathrm{c}}$ levels was associated with reductions in risk of $21 \%$ for any diabetes-related endpoint, $21 \%$ for diabetesrelated deaths, $14 \%$ for myocardial infarction and $37 \%$ for microvascular complications. There was no threshold for $\mathrm{HbA}_{1 \mathrm{c}}$ identified for risk reduction of any endpoint (Stratton 2000).

Thus, the risk of complications may be managed by maintaining $\mathrm{HbA}_{1 \mathrm{c}}$ within certain limits, which are usually tailored to the individual, and by considering the risks of hypoglycaemia as well as hyperglycaemia. For example, for most individuals, an $\mathrm{HbA}_{1 \mathrm{c}}$ level of $6.5-7.5 \%$ is desirable, whereas for those who are elderly or at risk of falls the targets may be rather looser. Pregnant women, on the other hand, require much tighter targets to minimise the risk of pregnancy complications.

Hypoglycaemic episodes (hypos) are a significant problem characterised by low glucose levels. Mild hypos are considered to be the price paid for good glycaemic control. However, severe hypos can result in loss of consciousness, seizures and even death.

One of the difficulties of living with diabetes is the delay in the development of the consequences 
of suboptimal glycaemic control: they do not occur immediately, unlike in conditions such as asthma or arthritis, where non-adherence has an immediate effect. It may take many years or even decades for diabetes complications to emerge, by which point it can be too late for them to be avoided or reversed. This chronological break makes it easier for patients not to prioritise diabetes and allows them to use denial as a defence mechanism for many years, while complications slowly develop.

Psychological factors are important in the maintenance of good glycaemic control. I will now examine the psychological barriers associated with the major psychiatric disorders, and proceed to some diabetes-specific psychological problems, such as fear of hypoglycaemia, fear of complications and specific difficulties relating to adherence to different aspects of diabetes management.

\section{Psychosis and diabetes}

People with mental health problems are at an increased risk of mortality, with a 'mortality gap' of over 10 years between people with and without psychiatric disorders (Lawrence 2013). People with psychotic illness carry an overall increased risk of cardiac death twice that of the general population, and diabetes is one of the contributing factors to this, as diabetes and the constellation of related risk factors (including insulin resistance, abdominal obesity, hypertension and lipid dysregulation) are much more common in severe mental illness (De Hert 2006; Mitchell 2012, 2013a). Type 2 diabetes is associated with excess mortality (twofold) in schizophrenia (Schoepf 2012). Vinogradova et al (2010) found increased mortality in diabetes in both schizophrenia and bipolar disorder and speculated that poorer glycaemic control may be a factor in this. In a Danish study that examined comorbidity of type 2 diabetes and psychosis, over one-third of natural deaths were attributed to diabetes, with $14 \%$ of these attributed to the interaction between diabetes and mental illness (Ribe 2014).

Psychotic disorders may create barriers to the management of diabetes in both the acute and the chronic phases of the disorders. In acute psychosis, the presence of behavioural disturbance and agitation maybeassociated with difficulties adhering to the complex self-management of diabetes. In chronic schizophrenia, negative symptoms and poor dietary choices present a challenge to optimal self-management, compounding the effects of antipsychotic medications (Newcomer 2005).

Incorporating lifestyle interventions as part of routine management of psychotic disorders improves health outcomes (Caemmerer 2012). The
Lester adaptation of the Positive Cardiometabolic Health Resource (Shiers 2014) provides evidencebased shared-treatment protocols for primary and secondary care. Psychiatrists and community mental health teams can play an important role in achieving and maintaining glycaemic control, as they often have far more intense patient contact than the diabetes services, and may be in a stronger position to drive lifestyle changes, perhaps by using a motivational interviewing approach.

\section{Depression and diabetes}

Depression is common (with a prevalence of about $10 \%$ ) in people who have poor physical health, particularly when they have experienced a recent major diagnosis or life-threatening event (Wulsin 2005). When present in diabetes, depression is associated with poor adherence to medications, physical inactivity, suboptimal glycaemic control, increased risk of diabetes complications, worse quality of life and increased use of unscheduled care (Lustman 2005; Ismail 2007). A study of patients with their first foot ulcer found that those who had any depressive symptoms at diagnosis had significantly higher mortality rates: they were 2-3 times more likely to have died by 18 -month follow-up (Ismail 2007).

The presence of a comorbid psychiatric disorder increases healthcare utilisation, the risk of longterm complications and overall cost burden. When diabetes care is integrated with the management of depression, biomedical as well as psychosocial outcomes improve (Katon 2004).

The diagnosis of major depression in people with chronic ill health can be complex, as the somatic symptoms of depression are frequently masked by symptoms attributable to the physical condition (Verhaak 2006; Parker 2010), although there is some evidence that even somatic symptoms may be distinguished (Mitchell 2013b). For this reason, there is limited evidence for the use of screening tools in diabetes for the detection of depression (Hermanns 2010). For example, somatic symptoms of anergia and insomnia may be attributed to persistent hyperglycaemia. Diabetes teams may be reluctant to approach the topic of mood or other psychological problems, particularly if the service does not have adequate liaison psychiatry expertise available (Coventry 2011).

\section{Eating disorders and diabetes}

Patients with type 1 diabetes have a twofold increased risk of eating disorders compared with the general population, with the prevalence ranging from $6.4 \%$ to $10.1 \%$ (Young 2013). A longitudinal study over 11 years of 207 women with type 1 
diabetes observed that the majority of those who used insulin restriction to control weight and shape at baseline continued to do so over time, and that the incidence of new-onset insulin restriction was high (23\%) (Goebel-Fabbri 2011).

The presentation of eating disorders in type 1 diabetes is different from their presentation in the general population, with insulin restriction (reduction/omission) to control weight a common symptom. This may be regarded as a form of purging. Although often suspected, it can be difficult to discuss with patients. Insulin restriction to control weight jeopardises diabetes management, leading to hyperglycaemia, increased risk of microvascular complications, repeated hospital admissions for diabetic ketoacidosis, earlier mortality and family stress (Jones 2000; Arcelus 2011). There are few intervention studies in this population and anecdotally they prove difficult to manage clinically. The only randomised controlled trial involving people with an eating disorder and type 1 diabetes found that, compared with standard care, basic psychoeducation improved eating symptoms but not glycaemic control or insulin omission, suggesting that expert diabetes guidance might be necessary for change in diabetes outcomes (Olmsted 2002).

\section{Anxiety disorders and diabetes}

Although depression is perhaps the most common mental disorder in diabetes, and eating disorders are arguably the most devastating, a wide variety of other mental disorders may have a significant impact on diabetes, its management and its prognosis. Anxiety disorders, especially needle phobia and its associated avoidance of insulin and blood glucose monitoring, can adversely affect self-management, as can obsessive-compulsive disorder, where there may be excessive checking of blood glucose. Studies have reported that $14 \%$ of people with diabetes met the criteria for generalised anxiety disorder and 40\% reported some anxiety symptoms (Grigsby 2002), and that anxiety symptoms were associated with poor glycaemic control (Anderson 2002). This relationship may be moderated by physical inactivity (Lipscombe 2014). Recent studies suggest that anxiety about glycaemic control may not be as common in diabetes as previously thought, although we must note that these studies used self-report questionnaires rather than diagnostic instruments (Strandberg 2014; Browne 2015).

Needle phobia is common in diabetes, in both type 1 and in insulin-treated type 2 (Mollema 2001; Cemeroglu 2014) and it is associated with poorer glycaemic control (Berlin 1997). It is also associated with higher rates of use of insulin pumps and poorer glycaemic control in pump therapy (Cemeroglu 2014). Needle phobia should be treated as other phobias, using a cognitivebehavioural approach involving relaxation and graded exposure - there is no evidence for any diabetes-specific treatments.

\section{Specific psychological problems in diabetes}

Diabetes is associated with specific psychological problems that may have a direct effect on glycaemic control and thus on the development of complications, future physical disability and mortality (Doherty 2014). Some complications of diabetes, in particular sexual problems, may also bring specific psychological difficulties. Box 1 shows a number of indications for psychiatric assessment in diabetes.

\section{Acceptance of diagnosis, denial, uncertainty}

Individuals who have received a recent diagnosis of diabetes frequently describe difficulties in adjusting to the diagnosis (Larsen 2014). In particular, they report difficulties accepting that they have a long-term condition that requires constant self-management if the long-term sequelae are to be avoided. This can involve a variety of responses, from a depressive reaction to denial and avoidance, all of which can result in poor glycaemic control.

\section{Fear of hypoglycaemia}

In addition to the serious physical risks associated with hypoglycaemia, a hypoglycaemic episode is an unpleasant subjective experience (Larsen 2014). This experience, which may be so severe as to require third-party assistance, loss of consciousness and even hospital admission, may make the individual so anxious to avoid it again

BOX 1 Indications for psychiatric evaluation in diabetes

- Evidence of depressed mood

- Low body mass index (BMI): this might indicate an eating disorder

- Reluctance to start insulin therapy: might indicate needle phobia or fear of insulin

- Persistent suboptimal glycaemic control

- Recurrent admissions (e.g. for diabetic ketoacidosis, recurrent severe hypoglycaemia)

- Difficulties at transition to adult services 
that they run their blood glucose much higher than recommended (often particularly at night) and as a result glycaemic control deteriorates.

\section{Fear of complications}

The potential complications of diabetes are usually explained to patients at diagnosis. Most patients take this information in their stride, but a small number become fearful of developing these conditions (Larsen 2014). Some individuals, on seeing a high glucose reading despite their best efforts, feel that no matter what steps they take, they cannot fully control their diabetes and immediately infer that this means that complications are inevitable. They feel discouraged about their diabetes control and perhaps avoid the necessary self-care activities (to avoid these feelings of failure). Paradoxically, this can, of course, increase the risk of developing diabetes complications. A cognitive-behavioural formulation of this is shown in Fig. 1.

\section{Adherence}

Most people with diabetes know what they need to do to manage the condition well, but some find it difficult to adhere to the full self-care regimen required for optimal diabetes control. This can be for any of a wide range of reasons, including mental illness and social problems that absorb the individual's attention. The demands of selfmanagement are great and can be difficult to incorporate into a busy lifestyle. For these self-care activities to occur, the individual must see them as important and prioritise them accordingly. A motivational interviewing approach can be useful

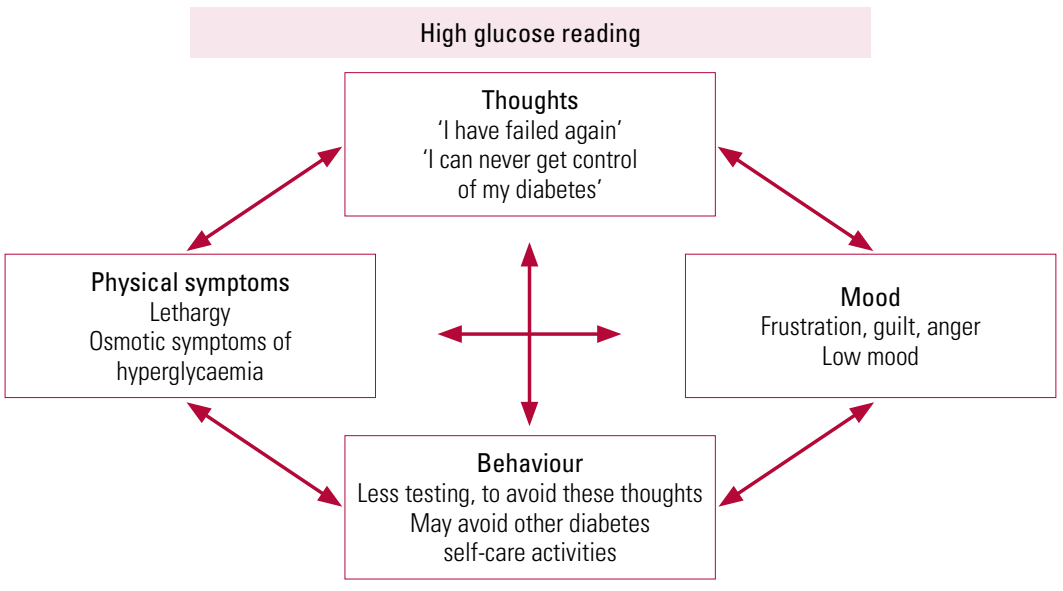

FIG 1 A cognitive-behavioural formulation of psychological reactions to an elevated blood glucose reading.

in helping patients to overcome the barriers to good self-care and to prioritise appropriately. This might be particularly important for young people with type 1 diabetes, among whom mortality is up to sixfold higher than in non-diabetic peers, partly attributed to psychological distress during transition into adult diabetes (Diabetes UK 2011). Figure 2 shows how adherence-related problems might be considered along a spectrum from general coping difficulties to mental illness, in a pyramid of need.

There is evidence that diabetes-focused psychological therapies, including motivational interviewing and cognitive-behavioural approaches, may be useful in improving adherence and glycaemic control (Katon 2004; Ismail 2010; McGregor 2011).

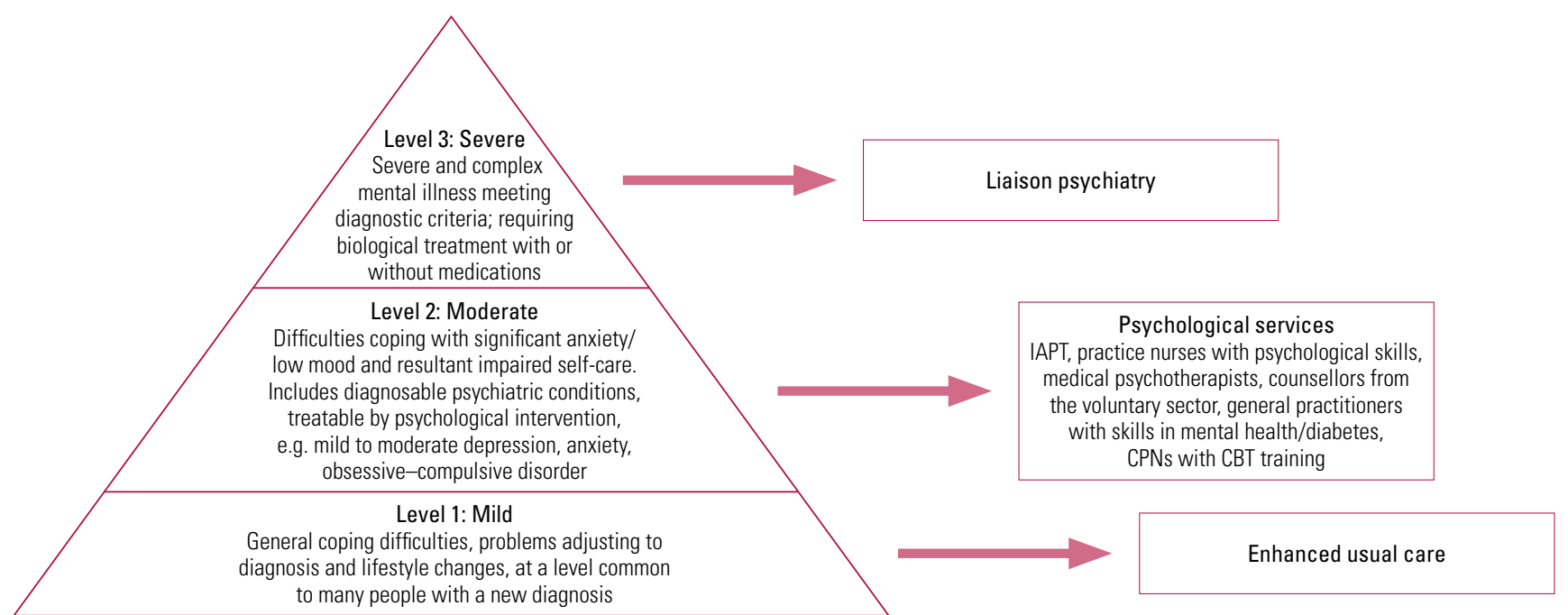

Pyramid of psychological problems in diabetes (adapted from Trigwell 2008 in Doherty 2013). CBT, cognitive-behavioural therapy; CPN, community psychiatric nurse; IAPT, Improving Access to Psychological Therapies programme. 


\section{Abnormal health beliefs}

Abnormal health beliefs regarding the use of insulin are common, especially in certain cultural groups (Larsen 2014). For example, in populations where there is a high prevalence of diabetes, individuals may have seen family members with advanced complications that coincided chronologically with insulin, and as a result may associate insulin with these complications. Stigma is also common in diabetes, with some patients reluctant to start insulin for fear that people will equate their injecting with intravenous drug use.

\section{The role of personality and coping}

The constellation of symptoms formerly known as brittle diabetes (severe glycaemic instability, with recurrent hospital admissions and early complications and mortality) are increasingly being recognised as having a significant psychological component. One recent study of the psychopathology of brittle diabetes found increased rates of maladaptive personality traits in individuals with this form of diabetes compared with those with stable diabetes (Pelizza 2014).

Social problems can likewise have an impact on diabetes and on general health (Saydah 2013). It seems intuitive that if someone is homeless, for example, this would be a much greater priority for them than the detail of diabetes self-care.

\section{The management of comorbid mental illness}

The treatment of axis I mental disorders in diabetes is not dissimilar to the management of these conditions in the general population. However, if the effect of diabetes on mental state is considered alongside the mental disorder, it is more likely that the individual's physical and mental health will improve. The effect of the mental disorder on the individual's physical health means that psychological interventions are indicated at a lower symptom threshold than in people who do not have diabetes.

When glycaemic control is rapidly improved, there is an increased risk of transient microvascular complications, especially retinopathy and neuropathy. It is advisable to request retinal photography in this instance.

\section{Pharmacological interventions}

Depression and anxiety

Antidepressants are frequently required for depression in diabetes and treatment is similar to that in the general population (NICE 2009). Certain antidepressants - duloxetine, amitriptyline and nortriptyline - are licensed for the treatment of pain due to diabetic neuropathy, and are useful choices for patients who have comorbid depression and neuropathic pain. Likewise, pregabalin is licensed for the treatment of neuropathic pain, and could be considered for comorbid neuropathy and anxiety.

The effect of antidepressants on glucose regulation is unclear. There have been case reports of both increased and decreased glucose associated with various antidepressant agents (Khoza 2011). Derijks et al (2008a) found that antidepressants were associated with a 1.5-fold increase in the risk of hyperglycaemia and a 1.8-fold increase in the risk of hypoglycaemia. A case-control study by the same authors showed that long-term antidepressant use (>3 years) in diabetes was associated with a 2.8-fold increase in the risk for severe hypoglycaemia (Derijks 2008b). Hypoglycaemia was associated with antidepressants with high affinity for serotonin reuptake transporters (i.e. selective serotonin reuptake inhibitors (SSRIS) and clomipramine), whereas hyperglycaemia was more strongly associated with those that have affinity for the $5-\mathrm{HT}_{2 \mathrm{c}}$ (serotonin) receptor, $\mathrm{H}_{1}$ (histamine) receptor, and noradrenaline reuptake transporters (i.e. amitriptyline, doxepin, imipramine, maprotiline, nortriptyline, mianserin, mirtazapine) (Derijks 2008a,b).

It had been postulated that antidepressants might act directly on reducing insulin resistance, although this has been refuted (Pyykkönen 2011).

\section{Psychosis}

Antipsychotics are a key component in the management of psychotic disorders. Many antipsychotic medications produce adverse metabolic effects, including weight gain (De Hert 2011) and increased incidence of diabetes mellitus (Correll 2015). A study in Austria and Germany of over 60000 individuals found that those taking antipsychotics had poorer glycaemic control and a higher rate of acute complications than those not taking them. They noted that, although atypical (second-generation) antipsychotics were associated with hyperglycaemia, there was no significant difference in hyperglycaemia between those on typical antipsychotics and those who took no antipsychotics (Galler 2015). Atypical antipsychotics are associated with up to three times the incidence of hyperglycaemia seen with typical antipsychotics; much of the increased risk is conferred by olanzapine and clozapine (De Hert 2008). Lipscombe et al (2011) found diabetes to be associated with both typical and atypical antipsychotics in elderly people. 
There is evidence that treatment with atypical antipsychotics may disrupt the neural activity associated with the anticipation and receipt of food rewards. Mathews et al (2012) report enhanced activation in the inferior frontal cortex, striatum and anterior cingulate cortex on anticipation of rewarding food, enhanced activation in the caudate and putamen on its receipt and decrease in responsivity to receipt of the rewarding food in the lateral orbital frontal cortex, an area thought to inhibit feeding behaviours. This brain activity is thought to be a likely mechanism for the development of type 2 diabetes in individuals taking antipsychotics. There is evidence that different atypical antipsychotics have varying effects on plasma glucose levels in mouse models (Savoy 2010), but this has not been replicated in humans.

The drug's metabolic profile is a key consideration in choosing an antipsychotic, but it must be balanced against a broader consideration of the health of the individual. In the case of refractory psychosis, the most effective drug, clozapine, has a very high rate of metabolic side-effects (Allison 1999; Le Noury 2008). In choosing an antipsychotic, these risks must be balanced against the evidence that in schizophrenia, those on clozapine live longest (with especially strong reductions in suicide rates), which suggests that the effective treatment of mental illness improves

\section{BOX 2 New models of care in diabetes and mental health}

\section{Dimensions of Care For Diabetes (3DFD)}

This model, developed in south London by a partnership between King's Health Partners and a number of London boroughs and community organisations, addresses the social and psychological dimensions of living with diabetes by integrating medical, psychological and social care for patients with persistent poor glycaemic control. This service has produced outcomes that demonstrate improved glycaemic control, psychological well-being and patient satisfaction, as well as reduced use of unscheduled care and its related costs (Archer 2012).

\section{TEAMcare}

TEAMcare evolved from a study by a multidisciplinary team from the University of Washington and the Group Health Research Institute (part of a nonprofit healthcare organisation in the USA). This new biopsychosocial intervention entails enhanced diabetes care for people with diabetes and depression, with psychological therapies delivered by diabetes nurses under the supervision of a psychiatrist (McGregor 2011). the individual's ability to manage both their physical and mental health (Tiihonen 2009).

There is emerging evidence that pharmacological strategies in schizophrenia, such as the use of metformin for weight loss on antipsychotics ( $\mathrm{Wu}$ 2008) and aripiprazole in addition to clozapine to promote metabolic health - it reduces weight and improves lipid profiles (Fleischhacker 2010) - may be effective in addressing the cardiovascular risk factors in this population.

\section{Non-pharmacological treatments}

Certain interventions, such as motivational interviewing and cognitive-behavioural therapy, have robust evidence for use in the management of psychological problems in diabetes (Ismail 2010).

Psychosocial interventions have been found effective for people taking antipsychotics in either delaying or reversing cardiovascular risk factors (Alvarez-Jimenez 2006; Caemmerer 2012).

Diabetes colleagues will likely welcome an opportunity to discuss shared patients with psychiatrists. Physicians are increasingly recognising the impact of mental health problems on patients' physical health and, in particular, on glycaemic control.

\section{Service considerations}

Diabetes is a common problem that frequently overlaps with mental illness. An awareness of the person's $\mathrm{HbA}_{1 \mathrm{c}}$ and supporting them to improve their risk profile, most specifically by reducing their $\mathrm{HbA}_{1 \mathrm{c}}$ by $1 \%$ (whether by dietary change, exercise or optimising management and adhering to it) will reduce their risk of developing the complications of diabetes.

The pyramid of need (Fig. 2) outlines which patients are likely to require enhanced usual care, which might need psychological input and which will require specialised psychiatry input.

A number of innovative models of combined care in diabetes and mental health have evolved in recent years, including the 3DFD model in the UK and the TEAMcare model in the USA (Box 2). These have demonstrated that the integration of psychiatry and diabetes care can result in improvements not only in patients' mental health, but also in their physical health (McGregor 2011; Archer 2012).

There is compelling evidence to show that addressing each individual's mental health and ensuring that, where possible, any psychiatric problems are treated and social needs are met, are key to improving the health and social functioning of people with diabetes. 


\section{References}

Allison DB, Mentore JL, Heo M, et al (1999) Antipsychotic-induced weight gain: a comprehensive research synthesis. American Journal of Psychiatry, 156: 1686-96.

Alvarez-Jimenez M, Gonzalez-Blanch C, Vazquez-Barquero JL, et al (2006) Attenuation of antipsychotic-induced weight gain with early behavioral intervention in drug-naive first-episode psychosis patients: a randomized controlled trial. Journal of Clinical Psychiatry, 67: 1253-60.

Anderson RJ, Grigsby AB, Freedland KE, et al (2002) Anxiety and poor glycemic control: a meta-analytic review of the literature. International Journal of Psychiatry in Medicine, 32: 235-47.

Arcelus J, Mitchell AJ, Wales J, et al (2011) Mortality rates in patients with anorexia nervosa and other eating disorders: a metaanalysis of 36 studies. Archives of General Psychiatry, 68: 724-31.

Archer N, Ismail K, Bridgen 0, et al (2012) Three dimensions of care for diabetes: a pilot service. Journal of Diabetes Nursing, 16: 123.

Berlin I, Bisserbe JC, Eiber R, et al (1997) Phobic symptoms, particularly the fear of blood and injury, are associated with poor glycemic control in type I diabetic adults. Diabetes Care, 20: 176-8.

Browne JL, Nefs G, Pouwer F, et al (2015) Depression, anxiety and self-care behaviours of young adults with Type 2 diabetes: results from the International Diabetes Management and Impact for Longterm Empowerment and Success (MILES) Study. Diabetic Medicine, 32: $133-40$

Caemmerer J, Correll CU, Maayan L (2012) Acute and maintenance effects of non-pharmacologic interventions for antipsychotic associated weight gain and metabolic abnormalities: a meta-analytic comparison of randomized controlled trials. Schizophrenia Research, 140: $159-68$

Cemeroglu AP, Can A, Davis AT, et al (2014) Fear of needles in children with type 1 diabetes mellitus on multiple daily injections (MDI) and continuous subcutaneous insulin infusion (CSII). Endocrine Practice, 6: 1-25.

Correll CU, Detraux J, De Lepeleire J, et al (2015) Effects of antipsychotics, antidepressants and mood stabilizers on risk for physical diseases in people with schizophrenia, depression and bipolar disorder. World Psychiatry, 14: 119-36.

Coventry PA, Hays R, Dickens C, et al (2011) Talking about depression: a qualitative study of barriers to managing depression in people with long term conditions in primary care. BMC Family Practice, 12: 10-17.

De Hert M, van Winkel R, Van Eyck D, et al (2006) Prevalence of diabetes, metabolic syndrome and metabolic abnormalities in schizophrenia over the course of the illness: a cross-sectional study. Clinical Practice and Epidemiology in Mental Health, 2: 14

De Hert M, Schreurs V, Sweers K, et al (2008) Typical and atypical antipsychotics differentially affect long-term incidence rates of the metabolic syndrome in first-episode patients with schizophrenia: a retrospective chart review. Schizophrenia Research, 101: 295-303.

De Hert M, Detraux J, van Winkel R, et al (2011) Metabolic and cardiovascular adverse effects associated with antipsychotic drugs. Nature Reviews Endocrinology, 8: 114-26.

Derijks HJ, Meyboom RH, Heerdink ER, et al (2008a) The association between antidepressant use and disturbances in glucose homeostasis: evidence from spontaneous reports. European Journal of Clinical Pharmacology, 64: 531-8.

Derijks HJ, Heerdink ER, De Koning FH, et al (2008b) The association between antidepressant use and hypoglycaemia in diabetic patients: a nested case-control study. Pharmacoepidemiology and Drug Safety, 17: 336-44.

Diabetes UK (2011) Diabetes in the UK 2011-2012: Key Statistics on Diabetes. Diabetes UK.
Diabetes UK (2014) Diabetes: Facts and Stats (Version 3, Revised: March 2014). Diabetes UK

Doherty A, Ismail K (2013) Commissioning Support and Implementing Best Practice Factsheet: Diabetes and Mental Health. Mental Health Training and Education Programme.

Doherty AM, Gaughran F (2014) The interface of physical and mental health. Social Psychiatry and Psychiatric Epidemiology, 49: 673-82.

Ewald N, Kaufmann C, Raspe A, et al (2012) Prevalence of diabetes mellitus secondary to pancreatic diseases (type 3c). Diabetes/ Metabolism Research and Reviews, 28: 338-42.

Fleischhacker WW, Heikkinen ME, Olie JP, et al (2010) Effects of adjunctive treatment with aripiprazole on body weight and clinical efficacy in schizophrenia patients treated with clozapine: a randomized, double-blind, placebo-controlled trial. International Journal of Neuropsychopharmacology, 13: 1115-25.

Galler A, Bollow E, Meusers M, et al (2015) Comparison of glycemic and metabolic control in youth with type 1 diabetes with and without antipsychotic medication: analysis from the nationwide German/ Austrian Diabetes Survey (DPV). Diabetes Care, 38: 1051-7.

Goebel-Fabbri AE, Anderson BJ, Fikkan J, et al (2011) Improvement and emergence of insulin restriction in women with type 1 diabetes. Diabetes Care, 34: 545-50.

Grigsby AB, Anderson RJ, Freedland KE, et al (2002) Prevalence of anxiety in adults with diabetes: a systematic review. Journal of Psychosomatic Research, 53: 1053-60.

Hermanns N, Kulzer B (2010) Screening in diabetes care: detecting and managing depression in diabetes. In Screening for Depression in Clinical Practice: An Evidence-Based Guide (eds AJ Mitchell, JC Coyne): 335-48. Oxford University Press.

Ismail K, Winkley K, Stahl D, et al (2007) A cohort study of people with diabetes and their first foot ulcer. Diabetes Care, 30: 1473-9.

Ismail K, Maissi E, Thomas S, et al (2010) A randomised controlled trial of cognitive behaviour therapy and motivational interviewing for people with type 1 diabetes mellitus with persistent sub-optimal glycaemic control: a Diabetes and Psychological Therapies (ADaPT) study. Health Technology Assessment, 14: 1-127.

Jones JM, Lawson ML, Daneman D, et al (2000) Eating disorders in adolescent females with and without type 1 diabetes: cross sectional study. BMJ, 320: 1563-6.

Katon WJ, Von Korff M, Lin EH, et al (2004) The Pathways Study: a randomized trial of collaborative care in patients with diabetes and depression. Archives of General Psychiatry, 61: 1042-9.

Khoza S, Barner JC (2011) Glucose dysregulation associated with antidepressant agents: an analysis of 17 published case reports. International Journal of Clinical Pharmacology, 33: 484-92.

Kropff J, Selwood MP, McCarthy MI, et al (2011) Prevalence of monogenic diabetes in young adults: a community-based, crosssectional study in Oxfordshire, UK. Diabetologia, 54: 1261-3.

Larsen T, Peyrot M, Rokne B (2014) Relationships of diabetesspecific emotional distress, depression, anxiety, and overall wellbeing with $\mathrm{HbA1C}$ in adult persons with type 1 diabetes. Journal of Psychosomatic Research, 77: 174-9.

Lawrence D, Hancock KJ, Kisely S (2013) The gap in life expectancy from preventable physical illness in psychiatric patients in Western Australia: retrospective analysis of population based registers. BMJ, 346: f2539.

Le Noury J, Khan A, Harris M, et al (2008) The incidence and prevalence of diabetes in patients with serious mental illness in North West Wales: two cohorts, 1875-1924 \& 1994-2006 compared. BMC Psychiatry, 8: 67 
Lipscombe LL, Lévesque LE, Gruneir A, et al (2011) Antipsychotic drugs and the risk of hyperglycemia in older adults without diabetes: a population-based observational study. American Journal of Geriatric Psychiatry, 19: 1026-33.

Lipscombe C, Smith KJ, Gariépy G, et al (2014) Gender differences in the relationship between anxiety symptoms and physical inactivity in a community-based sample of adults with type 2 diabetes. Canadian Journal of Diabetes, 38: 444-50.

Lustman PJ, Clouse RE (2005) Depression in diabetic patients: the relationship between mood and glycemic control. Journal of Diabetes Complications, 19: 113-22.

Mathews J, Newcomer JW, Mathews JR, et al (2012) Neural correlates of weight gain with olanzapine. Archives of General Psychiatry, 69: 1226-37.

McGregor M, Lin EH, Katon WJ (2011) TEAMcare: an integrated multicondition collaborative care program for chronic illnesses and depression. Journal of Ambulatory Care Management, 34: 152-62.

Mitchell AJ, Delaffon V, Vancampfort D, et al (2012) Guideline concordant monitoring of metabolic risk in people treated with antipsychotic medication: systematic review and meta-analysis of screening practices. Psychological Medicine, 42: 125-47.

Mitchell AJ, Vancampfort D, De Herdt A, et al (2013a) Is the prevalence of metabolic syndrome and metabolic abnormalities increased in early schizophrenia? A comparative meta-analysis of first episode, untreated and treated patients. Schizophrenia Bulletin, 39: 295-305.

Mitchell AJ, Ioannou N, Rampling JM, et al (2013b) Which symptoms are indicative of depression in epilepsy settings? An analysis of the diagnostic significance of somatic and non-somatic symptoms. Journal of Affective Disorders, 150: 861-7.

Mollema ED, Snoek FJ, Adèr HJ, et al (2001) Insulin-treated diabetes patients with fear of self-injecting or fear of self-testing: psychological comorbidity and general well-being. Journal of Psychosomatic Research, 51: 665-72.

Newcomer JW (2005) Second-generation (atypical) antipsychotics and metabolic effects: a comprehensive literature review. CNS Drugs; 19 (suppl 1): 1-93.

National Institute for Health and Care Excellence (2008a) Diabetes in Pregnancy: Management of Diabetes and its Complications from Pre-conception to the Postnatal Period (Clinical Guideline 63). NICE.

National Institute for Health and Care Excellence (2008b) Type 2 Diabetes (Clinical Guideline 66). NICE.

National Institute for Health and Care Excellence (2009) Depression in Adults with a Chronic Physical Health Problem: Treatment and Management (Clinical Guideline 91). NICE.

Olmsted MP, Daneman D, Rydall AC, et al (2002) The effects of psychoeducation on disturbed eating attitudes and behavior in young women with type 1 diabetes mellitus. International Journal of Eating Disorders, 32: 230-9.

Parker G, Hyett M (2010) Screening for depression in medical settings: are specific scales useful? In Screening for Depression in Clinical Practice: An Evidence-Based Guide (eds AJ Mitchell, JC Coyne): 191-202. Oxford University Press.

Pelizza L, Bonazzi F, Scaltriti S, et al (2014) Brittle diabetes: psychopathological aspects. Acta Biomedica, 85: 18-29.

Pyykkönen AJ, Raikkonen K, Tuomi T, et al (2011) Depressive symptoms, antidepressant medication use, and insulin resistance: the PPP-Botnia Study. Diabetes Care, 34: 2545-7.
Ribe AR, Laursen TM, Sandbaek A, et al (2014) Long-term mortality of persons with severe mental illness and diabetes: a population-based cohort study in Denmark. Psychological Medicine, 44: 3097-107.

Saydah SH, Imperatore G, Beckles GL (2013) Socioeconomic status and mortality: contribution of health care access and psychological distress among U.S. adults with diagnosed diabetes. Diabetes Care, 36: $49-55$.

Savoy YE, Ashton MA, Miller MW, et al (2010) Differential effects of various typical and atypical antipsychotics on plasma glucose and insulin levels in the mouse: evidence for the involvement of sympathetic regulation. Schizophrenia Bulletin, 36: 410-8.

Schoepf D, Potluri R, Uppal H, et al (2012) Type-2 diabetes mellitus in schizophrenia: increased prevalence and major risk factor of excess mortality in a naturalistic 7-year follow-up. European Psychiatry, 27: 33-42.

Shiers DE, Rafi I, Cooper SJ, et al (2014) Positive Cardiometabolic Health Resource: An Intervention Framework for People Experiencing Psychosis and Schizophrenia (Lester UK Adaptation, 2014 update). Royal College of Psychiatrists (http://www.rcpsych.ac.uk/PDF/ LesterUKAdaptation2014updateA5bookletversion.pdf).

Stenström G, Gottsäter A, Bakhtadze E, et al (2005) Latent autoimmune diabetes in adults definition, prevalence, $B$-cell function, and treatment. Diabetes, 54 (suppl 2): S68-72.

Strandberg RB, Graue M, Wentzel-Larsen et al (2014) Relationships of diabetes-specific emotional distress, depression, anxiety, and overall well-being with $\mathrm{HbA1C}$ in adult persons with type 1 diabetes. Journal of Psychosomatic Research, 77: 174-9.

Stratton IM, Adler Al, Neil HA, et al (2000) Association of glycaemia with macrovascular and microvascular complications of type 2 diabetes (UKPDS 35): prospective observational study. BMJ, 321: 405-12.

Tiihonen J, Lonnqvist J, Wahlbeck K, et al (2009) 11-year follow-up of mortality in patients with schizophrenia: a population-based cohort study (FIN11 study). Lancet, 374: 620-7.

Trigwell P, Taylor JP, Ismail K, et al (2008) Minding the Gap: The Provision of Psychological Support and Care for People with Diabetes in the UK. Diabetes UK.

UK Prospective Diabetes Study Group (1998) Intensive blood-glucose control with sulphonylureas or insulin compared with conventional treatment and risk of complications in patients with type 2 diabetes (UKPDS 33). Lancet, 352: 837-53.

van Bloemendaal L, Ten Kulve JS, la Fleur SE, et al (2014) Effects of glucagon-like peptide 1 on appetite and body weight: focus on the CNS. Journal of Endocrinology, 221: T1-16.

Verhaak PFM, Schellevis FG, Nuijen J, et al (2006) Patients with a psychiatric disorder in general practice: determinants of general practitioners' psychological diagnosis. General Hospital Psychiatry, 28: $125-32$

Vinogradova Y, Coupland C, Hippisley-Cox J, et al (2010) Effects of severe mental illness on survival of people with diabetes. British Journal of Psychiatry, 197: 272-7.

Wu RR, Zhao JP, Guo XF, et al (2008) Metformin addition attenuates olanzapine-induced weight gain in drug-naive first-episode schizophrenia patients: a double-blind, placebo-controlled study. American Journal of Psychiatry, 165: 352-8.

Wulsin LR, Evans JC, Vasan RS, et al (2005) Depressive symptoms, coronary heart disease, and overall mortality in the Framingham Heart Study. Psychosomatic Medicine, 67: 697-702.

Young V, Eiser C, Johnson B, et al (2013) Eating problems in adolescents with Type 1 diabetes: a systematic review with metaanalysis. Diabetes Medicine, 30: 189-98.

\section{$\mathrm{MCO}$ answers}

$\begin{array}{lllll}1 \mathrm{c} & 2 \mathrm{a} & 3 \mathrm{a} & 4 \mathrm{~b} & 5 \mathrm{a}\end{array}$ 


\section{MCQs}

Select the single best option for each question stem

1 A 24-year-old woman with a long history of type 1 diabetes diagnosed in early childhood is referred to the psychiatry service for assessment of mood. Her $\mathrm{HbA}_{1 \mathrm{c}}$ is $12.4 \%$ and has been above $9 \%$ for the past $3-4$ years. Her BMI is $19 \mathrm{~kg} / \mathrm{m}^{2}$. You decide that:

a she has good glycaemic control

b her glycaemic control is not important in your assessment

c a thorough assessment should include questions about her diabetes and her adherence to her regimen

$d$ it is not useful to obtain a collateral history from the diabetes clinic

e she has minimal risk of developing complications.

2 In considering this woman's diagnosis:

a eating disorder is an important differential diagnosis

b psychosis is a likely diagnosis

c depressive episode is unlikely

$\mathrm{d}$ dementia is likely

e fear of hypoglycaemia is rarely seen in patients with an elevated $\mathrm{HbA}_{1 \mathrm{c}}$.
3 You assess her and she tells you that she frequently omits insulin to manage her weight. She has just started a job in a prestigious media firm where her image is very important. You decide that:

a this is a common behaviour in young women with type 1 diabetes, but it must be addressed

b insulin omission rarely has long-term consequences

c NICE guidelines recommend watchful waiting $d$ there is a strong evidence base for the use of CBT in type 1 diabetes and eating disorder

e integrated diabetes care and psychiatric care are unlikely to be beneficial.

4 A 42-year-old man with a diagnosis of paranoid schizophrenia and type 2 diabetes treated with metformin has been admitted with a relapse of psychotic symptoms, secondary to non-adherence to oral antipsychotic medication. $\mathrm{His} \mathrm{HbA}_{1 \mathrm{c}}$ is $10.8 \%$. You decide that:

a type 2 diabetes carries limited risk of complications

b you need to ask about adherence to metformin and statins

c his $\mathrm{HbA}_{1 \mathrm{c}}$ will be an unreliable measure of glycaemic control if he has not been taking his medications $d$ he needs insulin

e reinstatement of metformin is unlikely to result in an improvement in glycaemic control.

5 On discharge 2 months later, his $\mathrm{HbA}_{1 \mathrm{c}}$ has improved to $8.4 \%$ but he reports blurred vision. You decide that:

$a$ it is important to ensure that he has retinal photography urgently

b continued improvement on discharge will not require lifestyle changes

c his community nurse or care coordinator has no role in encouraging lifestyle change

d smoking cessation will be important to minimise his cardiovascular risk

e his BMI is irrelevant. 